University of Massachusetts Amherst

ScholarWorks@UMass Amherst

1980

\title{
Analytical Aspects of Absorption Spectroelectrochemistry at a Platinum Electrode- II: Quantitative Basis and Study of Organic Compounds
}

Julian Tyson

University of Massachusetts Amherst

T. S. West

Macaulay Institute for Soil Research

Follow this and additional works at: https://scholarworks.umass.edu/chem_faculty_pubs

Part of the Chemistry Commons

\section{Recommended Citation}

Tyson, Julian and West, T. S., "Analytical Aspects of Absorption Spectroelectrochemistry at a Platinum Electrode- II: Quantitative Basis and Study of Organic Compounds" (1980). Talanta. 1280.

Retrieved from https://scholarworks.umass.edu/chem_faculty_pubs/1280 


\title{
ANALYTICAL ASPECTS OF ABSORPTION SPECTROELECTROCHEMISTRY AT A PLATINUM ELECTRODE-II
}

\section{QUANTITATIVE BASIS AND STUDY OF ORGANIC COMPOUNDS*}

\author{
J. F. TYSON
}

Department of Chemistry, University of Technology, Loughborough, U.K. and

T. S. WeST

Macaulay Institute for Soil Research, Craigiebuckler, Aberdeen, Scotland

(Received 3 September 1979. Accepted 4 October 1979)

\begin{abstract}
Summary-The theoretical basis for a new spectroelectrochemical technique, in which a narrow lightbeam is passed at grazing incidence over a plane electrode surface, has been derived. Agreement between theoretical and experimental behaviour has been obtained for a number of organic molecules with well-defined redox behaviour. The advantages of this technique over other spectroelectrochemical techniques are discussed with respect to potential applications in quantitative analysis and electrochemical studies.
\end{abstract}

The transient absorption phenomena observed close to the cathode during the electrolysis of dilute aqueous solutions of metal ions have been described. ${ }^{1-3}$ In a more recent paper, ${ }^{4}$ a full report of the dependence of the absorbance on experimental parameters was given. There was a linear dependence on concentration, showing the potential analytical usefulness of the effect. Furthermore, experiments were described and discussed, from the results of which it was deduced that the signals observed were due to the formation of hydroxo-complexes arising from interaction of the metal ions with hydroxide ions generated as a reduction product of surface platinum oxide, formed during the anodic pretreatment of the electrode.

Although, at that stage, the various effects observed had been satisfactorily accounted for in terms of the chemistry of the system, it was felt that to pursue the potential analytical usefulness, a proper theoretical quantitative basis was required. In view of the somewhat complex nature of the process occurring at the cathode, controlled by the kinetics of reduction of the surface platinum oxide as well as by the diffusion of hydroxide ions and metal ions in solution and the kinetics of their interaction, it was decided to investigate systems in which only diffusion and electrontransfer processes would be operative. Accordingly, reactions involving a single oxidized and single reduced species, both of which remain in solution, were studied, and results obtained with organic mol-

\footnotetext{
* Part of the experimental work described herein was carried out at Imperial College, London and part at the University of Aberdeen, Old Aberdeen, Scotland.
}

ecules of this type as the electroactive species are reported here. The quantitative relationship between absorbance and a number of experimental parameters is derived and discussed in the light of the results obtained, and in relation to the analytical potential of the system.

\section{THEORETICAL QUANTITATIVE BASIS}

The spectroelectrochemical arrangement is shown in Fig. 1a. It is assumed that (1) the electrode reaction being monitored is $\mathrm{Ox}+n \mathrm{e} \rightarrow \mathrm{Red}$, (2) that masstransport in the solution is by semi-infinite linear diffusion and (3) that only the species Red absorbs at the wavelength of observation.

\section{Absorbance measured normal to the electrode surfaces}

Consider first the situation shown in Fig. 1b, i.e. where the light-beam passes normally through an optically transparent electrode. The variation of absorbance with the appropriate experimental parameters has already been established for chronoamperometric conditions (i.e. the absorbance is monitored following a potential step).

The usual starting point for the derivation is the Cottrell equation ${ }^{5}$

$$
i_{1}=\frac{n F a D_{\mathrm{O}}^{1 / 2} C_{\mathrm{O}}^{\mathrm{b}}}{\pi^{1 / 2} t^{1 / 2}}
$$

where $i_{t}$ is the instantaneous current (A) flowing at time $t, n$ is the number of electrons transferred per mole, $a$ is the electrode area $\left(\mathrm{cm}^{2}\right), D_{0}$ is the diffusion coefficient of the oxidized species $\left(\mathrm{cm}^{2} / \mathrm{sec}\right), C_{\mathrm{O}}^{\mathrm{b}}$ is the bulk concentration of the oxidized species (mole/ml), $t$ 
(a)

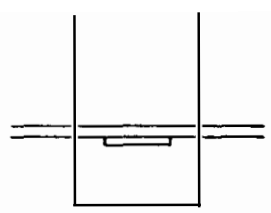

(b)

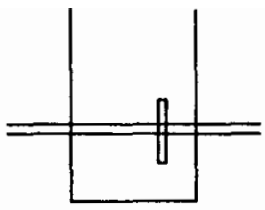

(c)

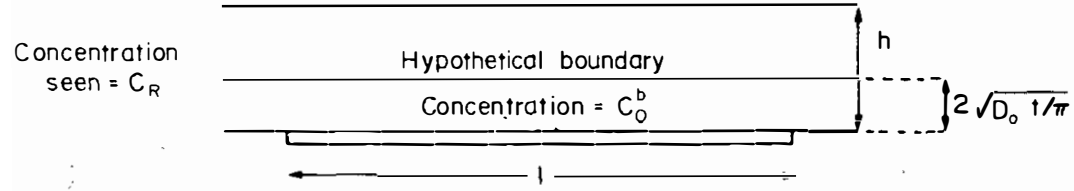

Fig. 1. Spectroelectrochemical configurations: (a) light-beam passing at grazing incidence over electrode: (b) light-beam passing normally through an optically transparent electrode; (c) hypothetical diffusion-layer behaviour.

is the time since the start of the electrolysis (sec), $F$ is the Faraday constant (coulombs), which is derived from a solution of Fick's laws of diffusion under the appropriate boundary conditions ${ }^{6.7}$ (one of which is that the concentration of the oxidized species is zero at the electrode surface during the electrolysis). Integration of equation (1) with respect to time gives the quantity of charge passed, $Q$, viz.

$$
Q=\frac{2 n F a D_{\mathrm{O}}^{1 / 2} C_{\mathrm{o}}^{\mathrm{b}} t^{1 / 2}}{\pi^{1 / 2}}
$$

From Faraday's laws, the amount of reduced species produced by the passage of $Q$ coulombs is $Q / F n$ moles. If the reduced species occupies a volume $V \mathrm{ml}$ then the concentration of the reduced species is given by

$$
C_{\mathrm{R}}=\frac{Q}{n F V}
$$

where the units of $C_{\mathrm{R}}$ are mole $/ \mathrm{ml}$.

The path-length of a beam of light passing normally through the electrode surface in the absorbing part of the solution will be $V / a \mathrm{~cm}$, and thus the absorbance will be given by

$$
A=\epsilon_{\mathrm{R}} \frac{V}{a} 1000 C_{\mathrm{R}}
$$

Substituting in equation (4) for $C_{\mathrm{R}}$ from equation (3) and for $Q$ from equation (2),

$$
A=\epsilon_{\mathrm{R}} 2\left(\frac{D_{\mathrm{O}} t}{\pi}\right)^{1 / 2} C_{\mathrm{O}}^{\mathrm{b}}
$$

where $C_{\mathrm{o}}^{\mathrm{b}}$ is now the bulk concentration of the oxidized species in mole/l.

Similar, though somewhat abbreviated, derivations have been given by Kuwana et al. ${ }^{8.9}$ In reference 8 , where this equation is first derived, the argument is confused by the use of the same symbol for electrode area and absorbance, and by not explaining the change in units of $C$. Equation (5) has also been derived by the method of Laplace transforms applied to the solution of Fick's equations (with the appropriate boundary conditions). ${ }^{10.11}$ In reference 10 , the unnecessary simplification is made of assuming the diffusion coefficients of the oxidized and reduced species to be equal. The equation has also been derived by integrating the appropriate function for $C_{\mathrm{R}}$ with respect to $x$, the distance from the electrode surface. ${ }^{12}$

$$
A=\epsilon_{\mathrm{R}} \int C_{\mathrm{R}} \mathrm{d} x
$$

and

$$
C_{\mathrm{R}}=C_{\mathrm{O}}^{\mathrm{b}} \operatorname{erfc}(z)
$$

where

$$
z=\frac{x}{2 D_{0}^{1 / 2} t^{1 / 2}}
$$

$\operatorname{Erfc}(z)$ is the error function complement of $z$ given by $\operatorname{erfc}(z)=1-\operatorname{erf}(z)$ where $\operatorname{erf}(z)$ is the error function of $z$ given by

$$
\operatorname{erf}(z)=\frac{2}{\pi^{1 / 2}} \int_{0}^{z} \mathrm{e}^{-y^{2}} \mathrm{~d} y
$$

This integral is evaluated numerically and can be found in standard tables.

At first sight, it may seem strange that the absorbance-time behaviour does not depend on the diffusion coefficient of the reduced species, the chemical entity actually being monitored. However, it must be remembered that the conditions in the diffusion layer are such that both concentration and path-length are changing and that, qualitatively speaking, if the reduced species is moving slowly the path-length will be short but the concentration high, and conversely if 
the reduced species is moving quickly then the pathlength will be long but the concentration low.

It can be seen from equation (5) that, as far as absorbance is concerned, the diffusion layer behaviour is exactly equivalent to a situation in which the concentration remains constant (at a value equal to the bulk concentration of the oxidized species) and the optical path-length is given by $2\left(D_{0} t / \pi\right)^{1 / 2}$.

\section{Absorbance measured parallel to the electrode surface}

Considering now the situation in Fig. 1a, where the absorbance parallel to the electrode surface, $A_{\mathrm{p}}$, is measured. If the height of the light-beam is $h$, then the concentration that the beam would detect, $C_{\mathrm{R}}$, is given by

$$
C_{\mathrm{R}} h=C_{\mathrm{O}}^{\mathrm{b}} 2\left(D_{\mathrm{o}} t / \pi\right)^{1 / 2}
$$

This is shown in Fig. 1c.

Now for the absorbance of the reduced form, $A_{\mathrm{pR}}=\epsilon_{\mathrm{R}} / C_{\mathrm{R}}$ where $l$ is the path-length on the electrode surface. Therefore

$$
A_{\mathrm{PR}}=\epsilon_{\mathrm{R}} \frac{12}{h}\left(D_{\mathrm{O}} t / \pi\right)^{1 / 2} C_{\mathrm{O}}^{\mathrm{b}}
$$

So far, only the reduced species has been considered as absorbing, but to account fully for the variation of absorbance with wavelength, the absorbance of the oxidized species must be taken into account. Although this aspect of the system has not been considered in any of the publications cited above it can be shown by arguments analogous to those presented for the reduced species that

$$
A_{\mathrm{po}}=A^{\prime}-\epsilon_{\mathrm{o}} \frac{2 l}{h}\left(\frac{D_{\mathrm{o}} t}{\pi}\right)^{1 / 2} C_{\mathrm{o}}^{\mathrm{b}}
$$

where $A^{\prime}$ is the initial absorbance and $\epsilon_{\mathrm{O}}$ is the molar absorptivity of the oxidized species. $A^{\prime}$ is, of course, given by $A^{\prime}=\epsilon_{\mathrm{O}} l C_{\mathrm{O}}^{\mathrm{b}}$.

Thus, in the experiments described here, the complete picture during electrolysis is that the absorbance is made up of three components, namely (1) the absorbance of the oxidized species outside the ends of the electrode, (2) the absorbance of the oxidized species within the path-length on the electrode surface and (3) the absorbance of the reduced species within the pathlength on the electrode surface.

If the cell path-length is $L \mathrm{~cm}$ then the total absorbance B

$$
\begin{aligned}
A_{\mathrm{p}_{\mathrm{T}}}= & \epsilon_{\mathrm{O}}(L-l) C_{\mathrm{O}}^{\mathrm{b}}+A^{\prime}-\epsilon_{\mathrm{O}} \frac{2 l}{h}\left(\frac{D_{\mathrm{O}} t}{\pi}\right)^{1 / 2} C_{\mathrm{O}}^{\mathrm{b}} \\
& +\epsilon_{\mathrm{R}} \frac{2 l}{h}\left(\frac{D_{\mathrm{O}} t}{\pi}\right)^{1 / 2} C_{\mathrm{O}}^{\mathrm{b}}=\epsilon_{\mathrm{O}} L C_{\mathrm{O}}^{\mathrm{b}}-\epsilon_{\mathrm{O}} l C_{\mathrm{O}}^{\mathrm{b}} \\
& +\epsilon_{\mathrm{O}} l C_{\mathrm{O}}^{\mathrm{b}}+\left(\epsilon_{\mathrm{R}}-\epsilon_{\mathrm{O}}\right) \frac{2 l}{h}\left(\frac{D_{\mathrm{O}} t}{\pi}\right)^{1 / 2} C_{\mathrm{O}}^{\mathrm{b}}
\end{aligned}
$$

In the experiments described here, the absorbance before electrolysis starts, $\epsilon_{\mathrm{O}} L C_{\mathrm{O}}^{\mathrm{b}}$, is set to zero, so

$$
A_{\mathrm{pr}}=\left(\epsilon_{\mathrm{R}}-\epsilon_{\mathrm{O}}\right) \frac{2 l}{h}\left(\frac{D_{\mathrm{o}} t}{\pi}\right)^{1 / 2} C_{\mathrm{O}}^{\mathrm{b}}
$$

Equation (7) is not quite the complete picture, as the absorbance will reach a maximum value when the "boundary" has diffused far enough from the electrode to fill the light-beam completely (see Fig. Ic), i.e., when

$$
2\left(\frac{D_{\mathrm{O}} t}{\pi}\right)^{1 / 2}=h
$$

Although equation (7) has been derived for a reduction at the electrode surface, the argument applies equally to an oxidation, when the resulting equation would be

$$
A_{\mathrm{pT}}=\left(\epsilon_{\mathrm{o}}-\epsilon_{\mathrm{R}}\right) \frac{2 l}{h}\left(\frac{D_{\mathrm{R}} t}{\pi}\right)^{1 / 2} C_{\mathrm{R}}^{\mathrm{b}}
$$

In deriving equation (7), no consideration has been given to longitudinal diffusion. This will only occur at the ends of the electrode, where there is a concentration gradient in a longitudinal direction. The occurrence of longitudinal diffusion will not affect the overall absorbance-time behaviour, as the number of absorbing centres in the light beam at any one time remains the same. The phenomenon has not been considered in previously reported electrochemical studies, as the regions of the solutions sampled by a light beam passing normally through an electrode contain a concentration gradient in only one direction.

\section{EXPERIMENTAL}

\section{Apparatus}

This was as described previously. ${ }^{4}$

\section{Reagents}

Three compounds were used: 4-amino-4'-methoxydiphenylamine (Variamine Blue), tris(5-nitro-1,10-phenanthroline)iron(II) perchlorate (nitroferroin) and 4,4'-diamino3,3'-dimethylbiphenyl (o-tolidine). The Variamine Blue was recrystallized before use. Analytical reagent grade potassium sulphate or sulphuric acid was used as background electrolyte.

\section{Procedure}

The function generator was preset to provide a step function so that the electrode in the light-path became either the cathode or the anode. A 5-ml portion of the working solution, the bulk of which was continuously deoxygenated with oxygen-free nitrogen, was transferred to the electrolysis cell and deoxygenated for a further $60 \mathrm{sec}$. During electrolysis the solution was kept under nitrogen. The initial transmission at the appropriate wavelength was set to $100 \%$ by adjusting the gain. The zero of the chartrecorder was set with the light-beam interrupted by an opaque material. The chart-recorder was started and the function generator was then switched to its preset value and the absorption signal recorded as a function of time, i.e., the experiments were the spectroelectrochemical analogue of chronoamperometry. The solution was finally 

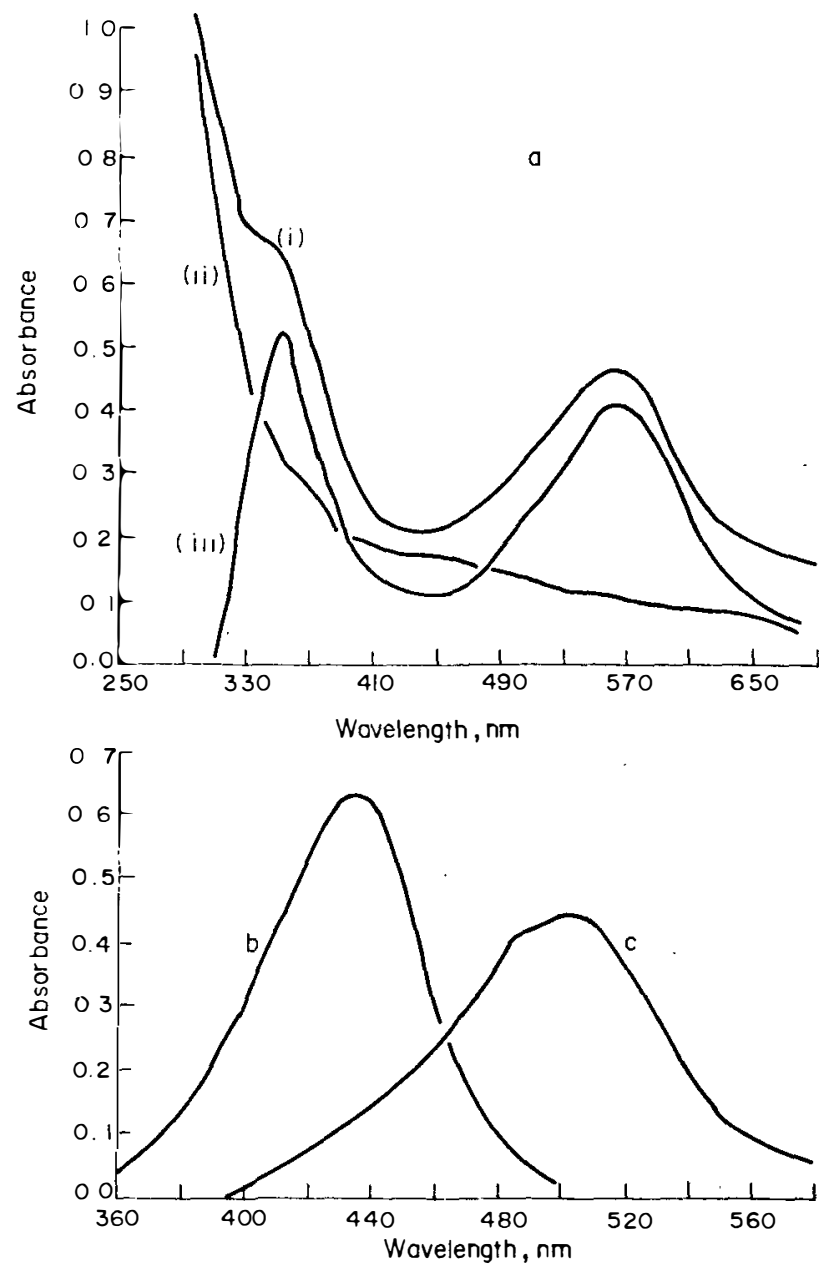

Fig. 5. Absorption spectra: (a) Variamine Blue; (i) oxidized form, (ii) reduced form, (iii) difference; (b) $o$-tolidine; (c) nitroferroin.

$o$-tolidine experiment the diffusion coefficient of nitroferroin is calculated to be $2.2 \times 10^{-6} \mathrm{~cm}^{2} / \mathrm{s}$.

\section{Variation of absorbance with wavelength}

The term in equation (8) governing the variation of absorbance with wavelength is the term including the molar-absorptivity difference. Thus the spectra obtained at the electrode surface should be the difference between the spectra of the oxidized and the reduced forms. This was verified by obtaining the spectra of the various compounds studied, with a double-beam spectrometer. The results are shown in Fig. 5. A comparison of these spectra with those in Fig. 3 shows excellent agreement between predicted and observed spectra, particularly in the case of Variamine Blue, the oxidized and reduced forms of which both absorb over the wavelength range studied. Care must be taken in interpreting the spectra obtained during spectroelectrochemical studies, as they may not arise from just one species.

\section{Variation of absorbance with concentration}

The theory predicts that there should be a linear relation between absorbance and the bulk concen- tration of the species diffusing towards the electrode, provided all other factors in the equation remain constant. This, of course, is the basis for the potential analytical usefulness of the technique. The analytical growth curves described earlier were obtained by recording absorbance as a function of time until a maximum value was reached, and plotting this absorbance maximum as a function of concentration. For o-tolidine, ten calibration points were recorded over the range $0-1.6 \times 10^{-5} \mathrm{M}$.

A least-squares fit of the best straight line yielded a slope of $5.6 \times 10^{4} \mathrm{l} . / \mathrm{mole}$, intercept $1.2 \times 10^{-3}$, correlation coefficient 0.99948 , and standard deviation of the scatter $9.2 \times 10^{-3}$. The theoretical slope was calculated to be $5.3 \times 10^{4} \mathrm{l} . / \mathrm{mole}$.

\section{Variation of absorbance with height of light-beam}

The theoretical $A v s$. $h$ plot is shown in Fig. 6 for $o$-tolidine at 10, 30 and $60 \mathrm{sec}$ after the start of the electrolysis. The experimental results in Fig. 4 show curves of a similar type though the agreement is not particularly good, most likely because of the difficulty of obtaining an accurate value for $h$ with the apparatus used. However, as already mentioned, in using 


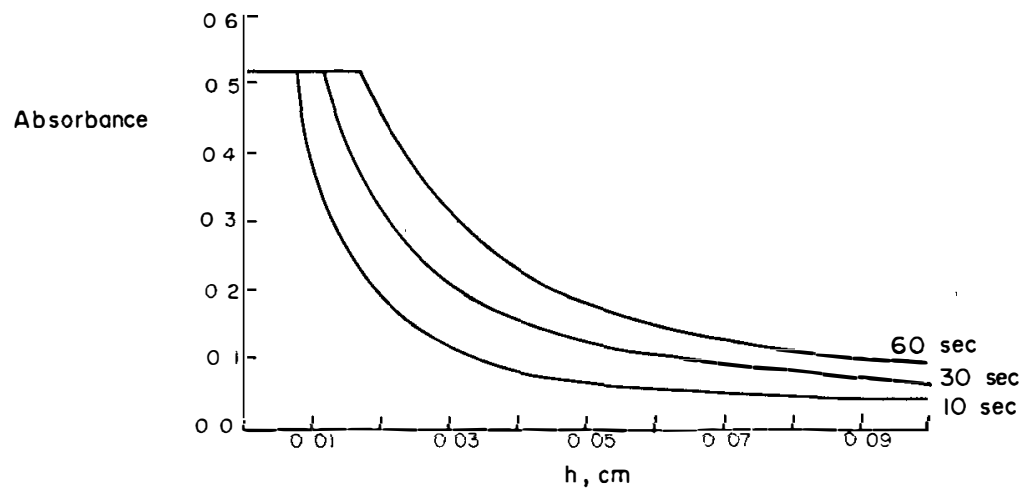

Fig. 6. Theoretical variation of absorbance of $o$-tolidine with the height of light-beam for different times after the start of the electrolysis.

the technique it is not necessary to know the individual values of $l$ and $h$, only their ratio, and this may be obtained from the electrolysis of a compound of known molar absorptivity and diffusion coefficient.

\section{Quantitative analysis}

\section{APPLICATIONS}

Spectroelectrochemical techniques have potential as quantitative analytical methods as the reaction conditions within the diffusion layer are highly reproducible and so problems associated (in conventional spectrophotometry) with the kinetics of the colourforming reaction, such as ensuring the colour is fully developed or is measured before it fades, are overcome. Obviously, the methods are only applicable to oxidation reduction systems, but subsequent reactions of electrolysis products may also be monitored, thus extending the possible scope. This is well illustrated by the example of the analytical growth curves obtained for the reaction of metal ions with hydroxide ions generated by the electroreduction of surface platinum oxide, reported previously. ${ }^{4}$ Existing spectroelectrochemical techniques have not found application in quantitative analysis to date; one of the reasons is probably the inherently poor sensitivity of existing methods, because of the very short pathlength in the absorbing medium.

With o-tolidine as a model compound, it has already been shown that for the method described here, the slope of the calibration curve is $5.5 \times 10^{4}$ l./mole (sensitivity $8 \times 10^{-8} M$ for $1 \%$ absorption) with an absorbance maximum reached after $125 \mathrm{sec}$. This may be compared with other spectroelectrochemical methods, as follows.

Internal reflection spectroscopy. The equation for the absorbance-time behaviour is ${ }^{15}$

$$
A=\epsilon b_{\text {cff }} C^{b}\left[1-\exp \left(a^{2} t\right) \operatorname{erf}\left(a t^{1 / 2}\right)\right]
$$

if the diffusion coefficients of the oxidized and reduced species are assumed to be equal. In this equation, $b_{\text {eff }}=\delta N_{\text {eff }}$ and $a=D^{1 / 2} / \delta$, where $\delta$ is the penetration depth which defines the optical path, and $N_{\text {eff }}$ is a sensitivity factor characteristic of the type of transparent electrode.
The time taken to reach the absorbance maximum, $\epsilon b_{\text {eff }} C^{b}$, is approximately $10^{-3} \mathrm{sec}$ for a $\delta$ value of $100 \mathrm{~nm}$. The slope of the calibration curve as calculated from results presented by Kuwana et al. ${ }^{16}$ for a tin oxide electrode, is $8.8 \mathrm{l} . / \mathrm{mole}$ (sensitivity $5.0 \times 10^{-4} M$ for $1 \%$ absorption).

Normal transmission through optically transparent electrodes in a thin-layer cell. For a thin-layer thickness of $10^{-3} \mathrm{~cm}$, complete electrolysis is achieved theoretically in $0.2 \sec ^{17}$ This would give rise to a calibration slope of $61 \quad$ l./mole (sensitivity $7.2 \times 10^{-5} \mathrm{M}$ for $1 \%$ absorption). This spectroelectrochemical study used a gold minigrid electrode ${ }^{\mathbf{1 8}}$ in a cell of thickness $8 \times 10^{-3} \mathrm{~cm}$. Taking the molar absorptivity of $o$-tolidine to be $6.1 \times 10^{4}$ 1. mole ${ }^{-1} \cdot \mathrm{cm}^{-1,13}$ the calibration slope would be $4.9 \times 10^{2} \mathrm{l} / \mathrm{mole}$ (sensitivity $9.0 \times 10^{-6} \mathrm{M}$ for $1 \%$ absorption). However, the results of the absorbance vs. time experiment ${ }^{18}$ presented give a value of $1.3 \times 10^{2} \mathrm{l}$./mole (sensitivity $3.5 \times 10^{-5} \mathrm{M}$ for $1 \%$ absorption), i.e., the molar absorptivity of the $o$-tolidine used was only $1.6 \times 10^{-4} 1 . \mathrm{mole}^{-1} \cdot \mathrm{cm}^{-1}$. The time taken to reach the maximum absorbance was approximately $15 \mathrm{sec}$. Nine years later, the experiment was reported as a teaching experiment. ${ }^{19}$ The cell thickness used was $1.7 \times 10^{-2} \mathrm{~cm}$, which should theoretically give a calibration slope of $1.0 \times 10^{3} \mathrm{l} / \mathrm{mole}$ (sensitivity $4.2 \times 10^{-6} \mathrm{M}$ for $1 \%$ absorption), but the absorbance results reported correspond to a value of $6.2 \times 10^{2} \mathrm{l} / \mathrm{mole}$ (sensitivity $7.1 \times 10^{-6} \mathrm{M}$ for $1 \%$ absorption) i.e., the $o$-tolidine used apparently had a molar absorptivity of $3.6 \times 10^{4}$ l.mole ${ }^{-1} . \mathrm{cm}^{-1}$ ). The absorbance-time behaviour was not reported. It should be pointed out that $o$-tolidine is carcinogenic, a fact not mentioned in the analytical literature.

Normal transmission through an optically transparent electrode. This experiment was carried out for $o$-tolidine at tin oxide optically transparent electrodes, ${ }^{8.9}$ but the absorbance-time behaviour was not reported. The relationship between absorbance and the experimental parameters is given by equation (5). and in theory the slope of the calibration curve can be made as large as required, as there is no limit to the 
path-length under "semi-infinite" conditions. However if a $1-\mathrm{cm}$ path-length is taken, the theoretical slope would be $6.2 \times 10^{4} \quad \mathrm{l}$./mole (sensitivity $7.1 \times 10^{-8} \mathrm{M}$ for $1 \%$ absorption) but the time taken to reach the maximum absorbance, given by $t_{\max }=h^{2} \pi / 4 D$, would be $2 \times 10^{5} \mathrm{sec}$.

Absorbance $v s$. time results have been reported for a gold minigrid electrode with two electroactive faces. ${ }^{20} \mathrm{~A}$ linear $A-t^{1 / 2}$ relationship is reported for $t$ between 1 and $10 \mathrm{sec}$. An absorbance of 1.0 is recorded after $10 \mathrm{sec}$, corresponding to a path-length of $0.02 \mathrm{~cm}$.

In this case, and also when the light-beam is parallel to the electrode surface, it is not necessary to wait until an absorption maximum is reached, as the bulk concentration is directly proportional to the slope of the $A$ vs. $t^{1 / 2}$ plot. Thus it is sufficient to record the absorbance-time behaviour until the slope of the $A v$ s. $t^{1 / 2}$ plot can be established, and then to obtain a calibration by plotting this slope against bulk concentration.

\section{Other applications}

Spectroelectrochemical techniques have been applied to the determination of a number of electrochemical parameters such as diffusion coefficients, rate constants, number of electrons transferred, $E^{0}$ values, and to the elucidation of reaction mechanisms. In addition to the advantage of high sensitivity, the technique described here (giving good signal to noise characteristics without the need for repetitive accumulation of data) has a number of other advantages. The light-beam does not pass through the electrode surface and thus there is no interference from the absorption spectrum of the electrode material, the usable wavelength range being determined by the transmission characteristics of the solvent. There will be no interference from non-Faradaic absorption processes and there is no limit to the type of electrode that may be used provided the surface does not become deformed during the experiment. The appropriate equations for more complicated electrode reactions may be readily derived by modifying the equations already derived in the literature to allow for the path-length on the electrode surface and the height of the light-beam. The speed of response of the system may be changed by changing the value of $h$, the lower limit being set by the spectrophotometer's ability to cope with low light-levels. The disadvantages of the technique may be that layers of solution very close (100 nm or so) to the electrode surface cannot be monitored and that deviations from the theoretical behaviour may occur owing to effects at the ends of the electrode.

Acknowledgements-One of us (JFT) gratefully acknowledges financial support from the Science Research Council and Technicon International.

\section{REFERENCES}

1. J. F. Tyson and T. S. West, Nature, 1974, 250, 139.

2. T. S. West, Analyst, 1974, 99, 886.

3. J. F. Tyson and T. S. West, Proc. Anal. Div. Chem. Soc. 1978, 15, 64.

4. Idem, Talanta. 1979, 20, 117.

5. Z. Galus, Fundamentals of Electrochemical Analysis p. 99. Horwood, Chichester, 1976.

6. P. Delahay, New Instrumental Methods in Electrochemistry, p. 46. Interscience, New York, 1954.

7. R. N. Adams, Electrochemistry at Solid Electrodes. p. 45. Dekker, New York, 1969.

8. J. W. Strojek and T. Kuwana, J. Electroanal. Chem. $1968,16,471$.

9. J. W. Strojek, T. Kuwana and S. W. Feldberg, J. Am. Chem. Soc., 1968, 90, 1353.

10. N. Winograd, H. N. Blount and T. Kuwana, J. Phjs. Chem., 1969, 73, 3456.

11. T. Kuwana and N. Winograd, in Electroanalyrical Chemistry Vol. 7, A. J. Bard, ed., p. 9. Dekker, New York, 1974.

12. J. F. Tyson, Ph.D. Thesis, University of London, 1975, p. 132.

13. Ref. 11, p. 53.

14. T. Kuwana, Ber. Bunsenges. Phys. Chem., 1973, 77, 858.

15. Ref. 11, p. 24.

16. W. N. Hansen, T. Kuwana and R. A. Osteryoung, Anal. Chem., 1966, 38, 1810.

17. C. N. Reilley, Rev. Pure Appl. Chem., 1968, 18, 137.

18. R. W. Murray, W. R. Heineman and G. W. O'Dom, Anal. Chem., 1967, 39, 1666.

19. T. P. DeAngelis and W. R. Heineman, J. Chem. Educ, 1976, 53, 594.

20. M. Petek, T. E. Neal and R. W. Murray, Anal. Chem, 1971, 43, 1069. 